\title{
Observation de la dynamique des dislocations - comparaison de différentes méthodes $\left(^{*}\right)$
}

\author{
L. P. Kubin \\ Laboratoire d’Optique Electronique, Laboratoire Propre du C.N.R.S. associé à IUniversité Paul Sabatier, Toulouse, \\ BP 4347, 31055 Toulouse Cedex, France
}

\begin{abstract}
Résumé. - La loi de vitesse d'une dislocation en fonction de la température et de la contrainte appliquée est examinée au moyen du formalisme de l'activation thermique. Divers résultats expérimentaux portant sur trois matériaux (cuivre, silicium et fer) et obtenus par différentes méthodes (essai de déformation, figures d'attaque, topographie aux rayons $\mathrm{X}$, microscopie électronique in situ) sont discutés dans ce cadre. Cette analyse fait ressortir l'intérêt qu'il y a à combiner plusieurs approches sur un même matériau lorsque l'on recherche une prévision des propriétés mécaniques à partir du comportement individuel des dislocations.
\end{abstract}

\begin{abstract}
The dependence of dislocation velocities on applied stress and temperature is examined within the framework of thermal activation. Experimental results obtained on three materials (copper, silicon and iron) and by different methods (straining experiments, etch-pits, X-ray topography and in situ electron microscopy) are discussed in this light. From such analysis, it appears how a better understanding of plasticity in terms of individual dislocation behaviour can be gained by combining several experimental methods applied to the same material.
\end{abstract}

1. Introduction. - Une dislocation se déplaçant par glissement dans un cristal doit franchir un spectre d'obstacles localisés et de force variable. Sa vitesse instantanée ou moyenne peut alors être exprimée en fonction de la température, $T$, et de la contrainte effective de cisaillement, $\tau$, qui lui est appliquée. Les propriétés mécaniques du cristal étudié résultent alors de la sommation, sur un volume macroscopique du matériau, des comportements individuels.

Différentes techniques permettent d'accéder de manière plus ou moins directe à la fonction $v=v(\tau, T)$ et dans les cas simples la théorie permet d'attribuer à celle-ci une forme précise. Dans ce qui suit nous considèrerons quatre de ces techniques : l'essai de déformation, l'étude des figures d'attaque, la topographie aux rayons $\mathrm{X}$ et la microscopie électronique in situ. Les deux dernières connaissent actuellement des développements qui permettent à l'expérimentateur d'aborder le problème des vélocités des dislocations sous son aspect microscopique et non plus seulement macroscopique. La possibilité de mener de front ces deux types d'approche est très prometteuse, mais il apparaît que chaque méthode a une tendance naturelle à édifier son propre langage et à évoluer séparé-

$\left.{ }^{*}\right)$ Conférence présentée au Congrès de la Société Française de Physique (Toulouse). ment, de telle sorte que le cloisonnement ainsi créé risque de nuire à une meilleure compréhension des mécanismes de plasticité.

Notre but sera ici de montrer l'unicité fondamentale des résultats obtenus par différentes approches, ou tout au moins d'illustrer quelques tentatives à travers les lois qui gouvernent les vitesses de dislocations par le jeu de l'activation thermique. Nous nous limiterons pour cela à des situations simples mettant en jeu le glissement des dislocations à travers un réseau d'obstacles localisés, dans une situation favorable (c'est le cas du fer) et dans deux situations plus complexes (c'est le cas du cuivre et du silicium).

Les deux premières parties sont consacrées à la loi de mobilité d'une dislocation telle que la décrit le formalisme de l'activation thermique et à la manière dont chacune des méthodes étudiées traduit cette loi sous forme accessible à l'expérimentation. Nous n'aborderons pas ici l'aspect technologique ni les caractéristiques physiques des images de dislocations obtenues dans chaque cas, qui sont relativement bien connus.

Les parties suivantes comparent les résultats obtenus sur un même matériau au moyen de ces diverses approches (le tableau I résume les références expérimentales correspondantes). Dans chaque cas, le problème central consiste à extraire de ces mesures une loi de vitesses et à lui attribuer un sens physique. 
Tableau I. - Répartition des références expérimentales discutées ci-dessous, suivant le matériau et l'approche utilisée.

[Distribution of the experimental work discussed below, following the methods and materials used.]

\section{Déformation}

Figures d'attaque

Topographie

Microscopie électronique
[4]

$[5,7,8]$

$\begin{array}{lcr}\mathrm{Cu} & \mathrm{Si} & \mathrm{Fe} \\ - & - & - \\ {[1]} & & {[5,6]} \\ {[1,2,3]} & \text { voir }[4] & \end{array}$

2. Loi de mobilité des dislocations dans le cadre de l'activation thermique. - Considérons une dislocation franchissant des obstacles séparés par une distance $\Lambda$, sous une contrainte de cisaillement $\tau$ et à la température $T$. Compte tenu de l'énergie d'activation $\Delta G(\tau)$ nécessaire pour le franchissement et qui caractérise l'obstacle, une loi de Boltzmann dicte la probabilité de passage par unité de temps sous l'effet des fluctuations thermiques, et donc le temps d'attente $\delta t$ :

$$
\delta t \sim\left(\mathrm{l}_{\mathrm{d}} \frac{b}{\Lambda}\right)^{-1} \exp \frac{\Delta G(\tau)}{k T}
$$

où tous les termes ont leur sens usuel. Entre deux obstacles la vitesse de la dislocation n'est limitée que par des effets de frottement visqueux du type interactions dynamiques dislocations-phonons, dislocationsélectrons, etc... Si l'on ne tient pas compte d'éventuels effets relativistes, on peut alors écrire :

$v$ (entre obstacles) $=\tau b / B$ où $B$ est la constante de frottement. La vitesse moyenne de la dislocation s'écrira alors, en combinant ces deux expressions :

$$
v=\frac{\Lambda}{\left(v_{\mathrm{d}} \frac{b}{\Lambda}\right)^{-1} \exp \frac{\Delta G(\tau)}{k T}+\left(\frac{\tau b}{B \Lambda}\right)^{-1}} .
$$

A température donnée et à forte contrainte (c'est-àdire à grande vitesse, typiquement au-delà du $\mathrm{ms}^{-1}$ ) l'énergie d'activation est très faible et c'est le terme de frottement visqueux qui prédomine. Aux faibles vitesses, l'expression (1) se réduit à :

$$
v=v_{0} \exp -\frac{\Delta G(\tau)}{k T}
$$

avec une vitesse limite $v_{0}=b v_{\mathrm{d}}$. La figure 1 est une transcription graphique de cette loi, en coordonnées $\log v, \tau$, et pour fixer les idées, les valeurs numériques utilisées correspondent sensiblement au cas du fer pur.

A très faible contrainte, l'énergie d'activation tend vers sa valeur maximale $\Delta G(0)$. Pour obtenir une vitesse qui s'annule avec la contrainte, il faut alors tenir compte de la probabilité de saut inverse de la dislocation, ce qui conduit à mettre l'expression (2)

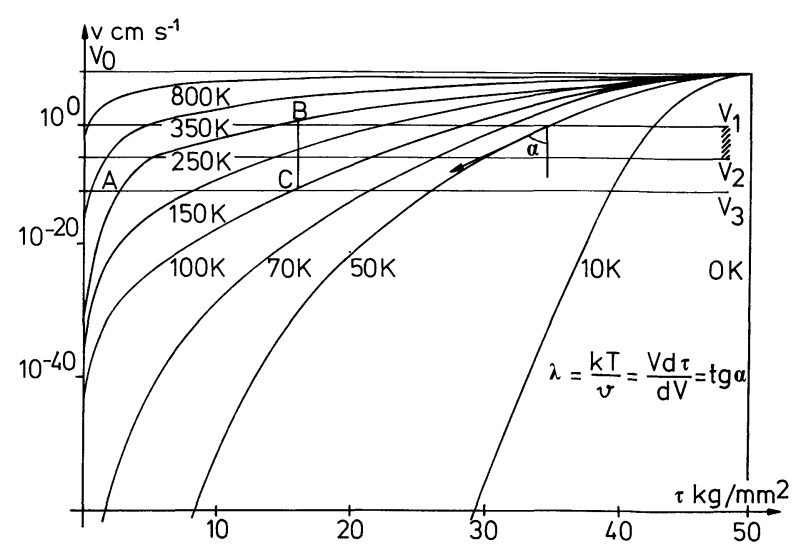

Fig. 1. - Vitesse d'une dislocation en fonction de la contrainte et de la température d'après le formalisme de l'activation thermique. Les valeurs numériques sont calculées pour un obstacle d'énergie d'activation $\Delta G=\Delta G_{0}\left(1-\tau / \tau_{0}\right)^{2}$ avec $\Delta G_{0}=0,75 \mathrm{eV}$ et

$$
\tau_{0}=50 \mathrm{~kg} / \mathrm{mm}^{2} \text {. }
$$

[Thermally activated velocity of a dislocation vs. stress at different temperatures. Numerical values are computed for an obstacle of activation energy $\Delta G=\Delta G_{0}\left(1-\tau / \tau_{0}\right)^{2}$, with $\Delta G_{0}=0.75 \mathrm{eV}$ and

$$
\left.\tau_{0}=50 \mathrm{~kg} / \mathrm{mm}^{2} .\right]
$$

sous forme d'un Sh. La figure 1 que nous discutons maintenant, tient compte de cette modification et donne un aperçu correct des vitesses de dislocations dans un large domaine de contraintes et de températures.

$\mathrm{Au}$ zéro absolu les dislocations sont immobiles tant que la contrainte n'atteint pas la valeur critique pour le franchissement de l'obstacle en l'absence d'activation thermique. A température non nulle, une vitesse non nulle est obtenue à plus faible contrainte. Cette vitesse varie typiquement de plusieurs ordres de grandeur pour une élévation de la contrainte de quelques $\mathrm{kg} / \mathrm{mm}^{2}$, ce qui explique l'apparition d'une limite élastique sur les courbes de déformation.

Les méthodes in situ ont, par coïncidence, approximativement accès au même domaine de vitesses que l'essai de déformation (de $10^{-2}$ à $10^{-7} \mathrm{~cm} \mathrm{~s}^{-1}$, c'està-dire entre $v_{1}$ et $v_{2}$ sur la figure 1 , tandis que $v_{3}$ représente le domaine des déformations géologiques : $10^{-12} \mathrm{~cm} \mathrm{~s}^{-1}$ ). Cependant, malgré l'exiguité de la lucarne expérimentale, l'équivalence entre température et vitesse de déformation permet de reconstruire le réseau de courbes au moyen d'un minimum de mesures. Par exemple, un changement de vitesse à température donnée (de $A$ à $B$ sur la figure 1) produit le même effet qu'un abaissement de température (de $150^{\circ}$ entre $A$ et $C$ ) à vitesse constante. On note ainsi sur la figure 1 que l'extrapolation des propriétés mécaniques à des vitesses de déformations géologiques est équivalente à un décalage en température compris entre 100 et $200^{\circ}$.

Un paramètre important et accessible à l'expérience est la sensibilité de la vitesse à une variation de contrainte. On utilise en général les quantités $\lambda=v \mathrm{~d} \tau / \mathrm{d} v$ 
(sensibilité à la vitesse de déformation) ou $V=k T / \lambda$ (volume d'activation) que l'on peut déduire de la figure 1 en considérant la pente $\operatorname{tg} \alpha=\mathrm{d} \tau / \mathrm{d} \log v$ des courbes $\log v=f(\tau)$ à $v$ donnée. On voit aisément que la variation de contrainte résultant d'un changement de vitesse $(\lambda)$ est nulle au zéro absolu puis augmente avec la température. Aux températures élevées elle présente un maximum puis devient très faible lorsque l'activation thermique efface les obstacles, de telle sorte qu'ils ne s'opposent plus au mouvement des dislocations (cf. Fig. 5, § 6). On dit alors que le mécanisme considéré devient athermique; notons cependant que en toute rigueur, il subsiste toujours un faible taux d'activation thermique, même aux plus hautes températures.

Enfin, le diagramme énergétique $\Delta G(\tau)$ de l'obstacle considéré peut être aisément reconstruit de la figure 1 à partir de l'expression (2) inversée :

$$
\Delta G(\tau)=k T \log v_{0} / v
$$

Du point de vue expérimental, le point à retenir est essentiellement le fait que dans l'hypothèse d'un seul type d'obstacle contrôlant la déformation, tous les points expérimentaux doivent se reporter sur une courbe unique $\Delta G(\tau)$ (ou $V(\tau)$ ) quelles que soient les températures ou les vitesses auxquelles ils ont été obtenus.

Ces diverses remarques sont illustrées plus loin par des exemples précis.

\section{Les traductions expérimentales de la loi de vitesses.} - Nous avons jusqu'ici considéré une dislocation isolée interagissant avec un seul type d'obstacle. Cependant les expériences à caractère microscopique nous montrent qu'aussi bien le comportement local des dislocations que les caractéristiques des obstacles sont sujets à d'importantes fluctuations. Celles-ci sont par ailleurs moyennées sur un grand nombre d'événements dans un essai de déformation à caractère macroscopique.

- La microscopie in situ permet, en principe, l'observation directe des vitesses de dislocations dans le domaine du $\mu \mathrm{m} \mathrm{s}^{-1}$, ou moins. L'hétérogénéité de la déformation à faible échelle constitue un facteur critique pour l'analyse de ces expériences. Pour cette raison, les cas les plus favorables concernent des matériaux contenant des obstacles forts et très denses, par exemple des forces de Peierls [9]. Le même problème se pose pour les méthodes de topographie aux rayons $\mathrm{X}$ dónt les progrès permettent d'envisager des observations quasi-continues du mouvement des dislocations (voir par exemple [10] pour une description d'expériences utilisant le rayonnement synchrotron, et [11] pour une revue des possibilités des diverses méthodes in situ). Les matériaux covalents, à forte force de Peierls et qui présentent des densités de dislocations raisonnables se prêtent particulièrement bien à l'étude par les méthodes de topographie.
- Par figures d'attaque, on atteint assez facilement des vitesses de l'ordre du $\mathrm{m} \mathrm{s}^{-1}$ mais cette technique comporte un certain nombre d'incertitudes dans la mesure où elle se situe à un niveau intermédiaire entre le microscopique et le macroscopique. Les mouvements de dislocations observés (typiquement sur plusieurs dizaines de microns) intègrent un grand nombre d'obstacles de natures diverses mais que l'on ne peut distinguer. Cette difficulté est illustrée plus loin par des expériences menées sur des alliages de cuivre. Au passage, il faut mentionner une tendance, née de l'observation de figures d'attaque, à reporter les vitesses de dislocations sur des diagrammes $\log v$, $\log \tau$ [12]. Ce procédé, valable à une époque où les lois de vitesses utilisées étaient encore empiriques, masque en fait les réalités physiques, et nous en donnerons deux exemples, l'un sur le cuivre et l'autre sur le fer.

- L'essai de déformation intègre les événements individuels sur de longs parcours et une densité de dislocations variable. Il présente donc l'avantage de conduire à l'événement moyen qui contrôle la déformation. Cet avantage est cependant compensé par sa très forte sensibilité aux effets de structure : multiplication, contraintes internes... qui ne sont pas directement accessibles.

La loi d'Arrhénius qui est couramment utilisée pour décrire la déformation plastique macroscopique représente en fait la sommation à l'échelle du cristal de l'éq. (2), à partir de la relation fondamentale :

$$
\stackrel{\circ}{\varepsilon}=\rho b v \text {. }
$$

On retiendra comme ordre de grandeur que si $\rho=$ quelque $10^{8} \mathrm{~cm}^{-2}$ et $b=$ quelque $10^{-8} \mathrm{~cm}$, $\stackrel{\circ}{\varepsilon}-10 v$ où $v$ est exprimée en $\mathrm{cm} \mathrm{s}^{-1}$. Dans l'hypothèse d'une structure constante, ou faiblement variable, les résultats d'un essai de déformation peuvent aisément être traduits sur un diagramme analogue à celui de la figure 1 , et inversement.

\section{Figures d'attaque et essais de déformation sur le} cuivre. - Meshii et Wada [1] ont étudié par figures d'attaque et essais de déformation la mobilité des dislocations dans du cuivre irradié aux électrons, et concluent que les deux méthodes aboutissent à des résultats cohérents. Ceci résulte probablement, et comme mentionné plus haut, de la présence dans le matériau étudié d'une densité suffisante de points d'ancrage efficaces. La figure 2 reproduit la loi de limite élastique du cuivre irradié obtenue par essai de déformation à vitesse constante, sur laquelle ont été reportées les contraintes nécessaires pour obtenir une vitesse de $10^{-3} \mathrm{~cm} \mathrm{~s}^{-1}$ par figures d'attaque. Le bon accord obtenu dans une large gamme de températures montre que, dans ce cas, l'événement moyen mesuré par l'essai de déformation a bien un sens physique précis au niveau d'une dislocation isolée. En reprenant les vitesses mesurées par figures 


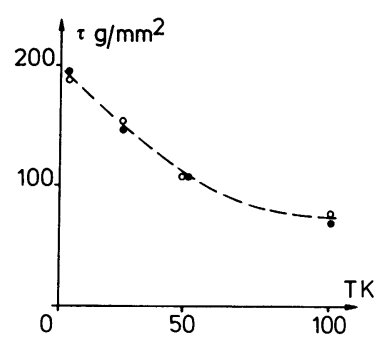

Fig. 2. - D'après Meshii et Wada [1] : comparaison d'essais de déformation à vitesse constante $(O$, limite élastique à

$$
\varepsilon=3 \times 10^{-4} \mathrm{~s}^{-1} \text { ) }
$$

et de mesures de vitesses par figures d'attaque (o, contrainte à $\left.v=10^{-3} \mathrm{~cm} \mathrm{~s}^{-1}\right)$.

[After Meshii and Wada [1] : comparison between constant strainrate experiments $\left(O\right.$, flow stress at $\left.\varepsilon=3 \times 10^{-4} \mathrm{~s}^{-1}\right)$ and etch-pit studies $\left(\Theta\right.$, stress at $\left.v=10^{-3} \mathrm{~cm} \mathrm{~s}^{-1}\right)$.]

d'attaque, il est possible de calculer le volume d'activation correspondant qui se trouve représenté sur la figure 3. Bien qu'obtenus sur une gamme assez large de vitesses $\left(10^{-1}\right.$ à $\left.10^{-6} \mathrm{~cm} \mathrm{~s}^{-1}\right)$ et de températures $(4,2-100 \mathrm{~K})$ les points expérimentaux se regroupent sur une seule courbe d'où l'on peut évaluer à environ $60 \mathrm{~g} / \mathrm{mm}^{2}$ la valeur de la composante athermique de la contrainte.

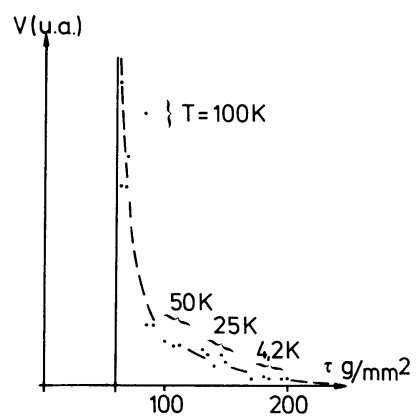

Fig. 3. - Volume d'activation calculé d'après les résultats de Meshii et Wada [1].

[Activation volume computed from the results of Meshii and Wada [1].]

Cette situation favorable n'est cependant pas la règle comme le montrent les expériences menées par Kleintges et al. [2, 3] sur des alliages de cuivre-aluminium $(0,31$ et $0,69 \%)$. Dans ce cas des créneaux de contrainte sont appliqués pendant des durées de l'ordre de quelques dizaines de microsecondes, entre lesquels des examens de figures d'attaque sont effectués. Le déplacement correspondant des dislocations est de l'ordre de $50 \mu \mathrm{m}$, ce qui correspond au franchissement d'environ 5000 obstacles substitutionnels d'aluminium. La vitesse des dislocations est de l'ordre de $1 \mathrm{~m} \mathrm{~s}^{-1}$ et les auteurs tiennent compte d'un terme de friction dont la valeur est évaluée à
$B / b=11,78 \mathrm{~N} \mathrm{~cm}^{-2} / \mathrm{cm} \mathrm{s}$ à $300 \mathrm{~K}$ en bon accord avec d'autres estimations des interactions dislocationsphonons.

L'étude des histogrammes - distance parcourue en fonction de la contrainte et de son temps d'application - révèle toutefois une très large dispersion. Il apparaît que les dislocations rencontrent des obstacles forts, peut être des amas de soluté, tous les $20 \mu \mathrm{m}$ avec une probabilité non négligeable d'y être bloquées. Néanmoins et ne tenant compte que des dislocations ayant une vitesse moyenne élevée, les auteurs arrivent à un accord raisonnable avec les prédictions de la théorie statistique de Labusch (cf. [3] pour plus de détails).

Le manque de concordance entre ces résultats traduits en termes d'essai de déformation et d'autres mesures par cette dernière méthode est attribué aux obstacles forts dont la contribution a été négligée. Cette étude par ailleurs très détaillée illustre bien la complexité de la déformation à faible échelle, ainsi que la difficulté de l'analyse dans ce cas.

\section{Topographies $X$ et figures d'attaque. Le cas du} silicium. - La mise au point d'une loi de vitesses pour le silicium se heurte à des difficultés tant théoriques qu'expérimentales. Si l'on ne considère que le silicium intrinsèque, il est probable que les forces de Peierls contrôlent la déformation, les modèles devant toutefois tenir compte d'une faible dissociation. Le tableau II d'après George [4] reproduit les résultats de diverses tentatives expérimentales afin d'établir une loi de vitesse de la forme :

$$
v \alpha \tau^{m} \exp -\frac{\Delta G(\tau)}{k T}
$$

Ces résultats appellent plusieurs remarques : les expériences menées à une seule contrainte conduisent à $m=0$ et $\Delta G=$ Cte. Ceci est normal compte tenu de ce que la lucarne expérimentale est alors très étroite : $650-800^{\circ}$ correspondent en effet à 0,46 et $0,57 T_{\mathrm{F}}$ respectivement ( $T_{\mathrm{F}}$, température de fusion). Dans un domaine plus vaste de contraintes il semble qu'il y ait accord sur une valeur de $m$ un peu supérieure à 1 et une énergie d'activation qui dépend de la contrainte. Dans ce cas, ainsi que l'explique George [4], l'identification avec un modèle de Peierls dépend de la nature des obstacles qui contrôlent la mobilité latérale des décrochements sur les dislocations vis. Un bon accord serait obtenu, par exemple, pour des obstacles dont l'espacement le long des lignes de dislocations serait de l'ordre du $\mu \mathrm{m}$. Les premières expériences par microscopie électronique in situ [13] n'ont pas encore permis de mettre en évidence leur nature.

6. Essais de déformation et microscopie électronique : le cas du fer. - Dans l'exemple présent, la géométrie particulière du cœur des dislocations induit une forte force de friction de réseau. En dépit de la diffi- 
Tableau II. - D'après George. Paramètres expérimentaux de la loi de vitesses dans le silicium suivant divers auteurs référencés en [4].

[After George. Experimental values for the parameters involved in the velocity law of silicon. The corresponding references are given in [4].]

$\begin{array}{ccc}\begin{array}{c}\text { Technique } \\ \text { d'observation }\end{array} & \begin{array}{c}\text { Contrainte } \\ (\mathrm{MPa})\end{array} & \begin{array}{c}\text { Température } \\ \left({ }^{\circ} \mathrm{C}\right)\end{array} \\ - & - & - \\ & 6-60 & 600-940\end{array}$

Figures d'attaque

$\begin{array}{cc}5-200 & 450-800 \\ 10-400 & 600-800 \\ 0,36 & 775-920 \\ 1-30 & 520-800 \\ 6-24 & 600-800 \\ 22 & 650-800\end{array}$

culté du problème à l'échelle atomique, son aspect énergétique est bien connu et la loi de vitesse des dislocations est bien comprise à partir d'essais de déformation et de diverses observations par microscopie électronique. Nous illustrerons donc au moyen de trois séries d'expériences les caractéristiques de la loi de vitesses discutée au $\S 2$.

Mughrabi et al. [5, 14] étudiant la déformation cyclique du fer à $300 \mathrm{~K}$ en fonction de la vitesse de déformation (de $10^{-2}$ à $10^{-5} \mathrm{~s}^{-1}$ ) arrivent à la conclusion suivante : à forte vitesse de déformation, une forte contrainte thermique contrôle la déformation, et une faible contrainte athermique à faible vitesse. Ceci est interprété [14] comme résultant de l'équivalence entre vitesse de déformation et température : lorsque la vitesse imposée aux dislocations est forte, la probabilité de franchissement des forces de friction de réseau doit être maintenue par une forte contrainte ce qui équivaut à un régime de basses températures. Dans le cas contraire, les temps d'attente imposés sont suffisamment longs pour que les fluctuations thermiques assurent le franchissement. Ce sont alors les interactions athermiques entre dislocations qui constitueront l'obstacle majeur au mouvement. L'observation de lames minces par microscopie électronique [5] confirme cette interprétation (cf. Fig. $4 a$ et $b)$ : sur la figure $4 a\left(\varepsilon=10^{-2} \mathrm{~s}^{-1}\right)$ les dislocations apparaissent bloquées en position vis par les forces de réseau, tandis que sur la figure $4 b$ $\left(\varepsilon=2,5 \times 10^{-5} \mathrm{~s}^{-1}\right)$ la présence de multipôles coin atteste le caractère athermique de la déformation.

La figure 5, d'après Peyrade [6] représente la sensibilité à la vitesse de déformation de fer pur et de fer dopé avec $560 \mathrm{ppm}$ atomiques de carbone (dont 360 dissous). La courbe du fer pur présente bien l'allure prévue d'après la loi de vitesses de la figure 1. En

\begin{tabular}{cccc}
\multicolumn{2}{c}{ Dislocations à $60^{\circ}$} & \multicolumn{2}{c}{ Dislocations vis } \\
$m$ & $\Delta G_{\mathrm{ev}}$ & $m$ & $\Delta G_{\mathrm{ev}}$ \\
- & - & - & - \\
1,4 & 2,2 & & 2 \\
1,4 & 2 & 1,4 & 2 \\
1,5 & $1-2$ & 1,5 & $1-2$ \\
& & 1,5 & 2,4 \\
& & 1,8 & 1,8 \\
$0,9 \rightarrow 1,45$ & $2 \rightarrow 2,7$ & & 2,2 \\
$\mathrm{~T} \downarrow$ & $\tau \downarrow$ & 1,1 & $2,5 \rightarrow 2,1$ \\
& $2,3 \rightarrow 1,9$ & & $\tau \downarrow$ \\
& $\tau \downarrow$ & & 2,1
\end{tabular}

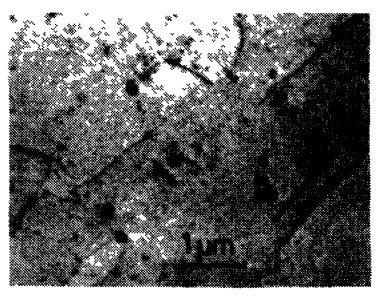

a)

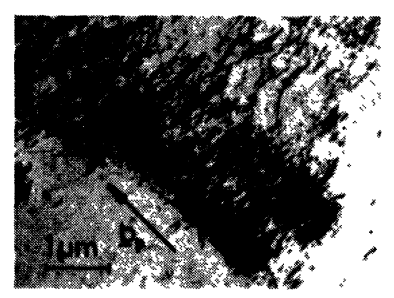

b)
Fig. 4. - D'après Mughrabi et al. [5]. Monocristaux de fer observés par microscopie électronique après une déformation cyclique

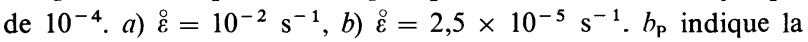
direction vis : $\langle 111\rangle$.

[After Mughrabi et al. [5]. Electron microscopy observation of iron single crystals after $10^{-4}$ of plastic strain of $\varepsilon=10^{-2} \mathrm{~s}^{-1}$. b) $\stackrel{\circ}{\varepsilon}=2.5 \times 10^{-5} \mathrm{~s}^{-1} . b_{\mathrm{P}}$ is along the screw direction : $\langle 111\rangle$.]

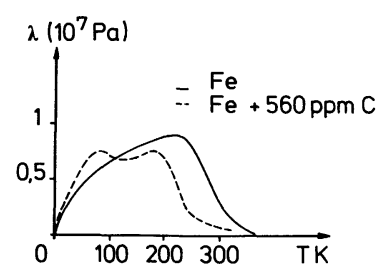

Fig. 5. - D'après Peyrade [6] variation avec la température du coefficient de sensibilité à la vitesse de déformation dans le fer pur et le fer dopé au carbone à la limite élastique. $\left(\stackrel{0}{\varepsilon}=7 \times 10^{-5} \mathrm{~s}^{-1}\right.$.) [After Peyrade [6] strain-rate sensitivity $v$ s. temperature for pure and carbon-doped iron at the flow stress. $\left(\dot{\varepsilon}=7 \times 10^{-5} \mathrm{~s}^{-1}\right)$.]

revanche la courbe du fer dopé présente deux maxima et selon toute probabilité deux mécanismes concurrents contrôlent la déformation, l'un étant sans doute 
le même que dans le cas du fer pur. Ce point est confirmé par l'observation in situ en microscopie électronique de ces mêmes échantillons [7] qui met en évidence l'existence de deux temps d'attente très différents. L'un correspond à la formation de double décrochements dans un réseau de fer pur (énergie d'activation $\sim 0,75 \mathrm{eV}$ ) et l'autre à leur formation au voisinage d'atomes de carbone (énergie d'activation $\sim 0,5 \mathrm{eV})$.

La loi de vitesses construite sur cette hypothèse rend alors bien compte de tous les résultats obtenus par essais de déformation [15].

La figure 6 montre une autre tentative de corrélation entre essai de déformation et examen in situ : Saka et al. [8] ont mesuré in situ à 180 et $300 \mathrm{~K}$ la vitesse de dislocations vis dans le fer (entre $10^{-3}$ et $10^{-5} \mathrm{~cm} \mathrm{~s}^{-1}$ )

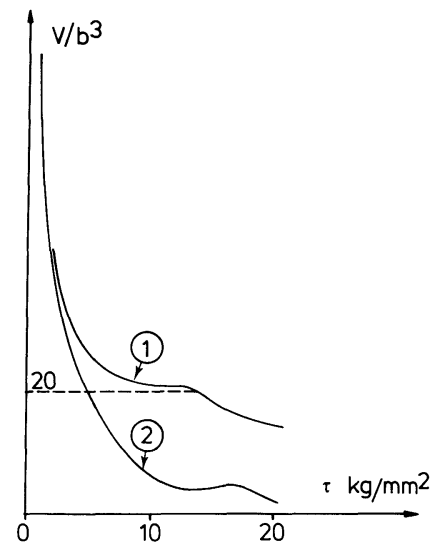

Fig. 6. - Comparaison de volumes d'activation dans le fer 1) D'après Peyrade [6], par essais de déformation. 2) Calculé d'après les mesures in situ de Saka et al. [8], à une constante arbitraire près.

[Comparison of activation volumes in iron by two methods. 1) After strain-rate experiments of Peyrade [6]. 2) Computed from the results of Saka et al. [8] (arbitrary units).] en fonction de la contrainte. Leurs résultats sont présentés ici transcrits sous la forme de volumes d'activation (en raison de larges incertitudes systématiques dues au mode de mesure les unités portées sur l'axe des ordonnées sont arbitraires), et comparés à ceux de Peyrade [6], obtenus par essais de déformation entre 35 et $300 \mathrm{~K}$ et à des vitesses de déformation de l'ordre de $10^{-4} \mathrm{~s}^{-1}$. On remarque sur les deux courbes l'apparition d'une bosse aux fortes contraintes, et dont l'interprétation n'est pas à ce jour certaine. Son existence au niveau de la dislocation isolée (par mesures in situ) semblerait cependant indiquer une nature intrinsèque, c'est-à-dire liée aux forces de friction.

7. Conclusion. - L'établissement d'un lien entre propriétés mécaniques et propriétés des dislocations requiert la mise en œuvre de deux stades intermédiaires : l'un consiste à connaître et à utiliser un formalisme décrivant les vitesses des dislocations, et possédant des bases physiques qui en permettent l'exploitation. Les quelques exemples mentionnés ci-dessus nous montrent que ce formalisme est relativement simple, même si la nature des interactions mises en jeu, c'est-à-dire l'énergie d'activation, n'est pas toujours aisément prévisible par le calcul. Le second stade revient à sommer la loi de vitesses sur une population de dislocations de densité variable, soumises à des contraintes internes, et devant franchir un spectre plus ou moins large d'obstacles. Suivant le matériau utilisé et la température cet aspect de la déformation prédomine ou au contraire disparaît complètement. Quelle que soit la situation expérimentale, deux nécessités semblent apparaître en vue de progrès futurs : d'une part celle qui consiste à combiner des approches mettant en jeu divers niveaux de résolution, et d'autre part celle de conserver dans ce cas une conception unitaire de ce qu'est la vitesse d'une dislocation.

\section{Bibliographie}

[1] MeShiI, M., WADA, M., In Proceedings of ICSMA 4 (ENSMIM, INPL Nancy, France) 1976, vol. 2, p. 819.

[2] Kleintges, M., Labusch, R., Brion, H. G., Haasen, P., Acta Metall. 25 (1977) 1247.

[3] Ney, H., Labusch, R., HaAš́ñ , P., Acta Metall. 25 (1977) 1257.

[4] GeORge, A., Thèse INPL, Nancy, 1977.

[5] Mughrabi, H., Herz, K., Ackermann, F., In Proceedings of ICSMA 4 (ENSMIM, INPL, Nancy, France) 1976, vol. 3 , p. 1244.

[6] Peyrade, J. P., Thèse Université Paul Sabatier, Toulouse, 1978.

[7] Kubin, L. P., Louchet, F., Acta Metall. 27 (1979) 337.

[8] Saka, H., Noda, K., Imura, T., Cryst. Lattice Defects 4 (1973) 45.

[9] Kubin, L. P., 21e Colloque de l'A.V.C.P. (E.P.F. Lausanne) 1979.
[10] Miltat, J., In Synchrotron radiation instrumentation and new developments. Ed. F. Wuilleumier et Y. Farge (North. Holland) 1977 , p. 323.

[11] Joufrrey, B., Proc. Int. Symposium on HVEM in situ experiments. Halle RDA, 1979. A paraître dans Phys. Status Solidi.

[12] Gilman, J. J., Micromechanics of flow in solids (Mc GrawHill) 1969 , p. 178.

[13] Sato, M., Sumino, K., In HVEM 1977. Japanese Society for electron microscopy, Ed. T. Imura et $\mathrm{H}$. Hashimoto 1977, p. 459.

[14] Herz, K., Mughrabi, H., Wilkens, M., Arbeitsbericht, MPI 177/P3 (Max-Planck Institut Stuttgart RFA), 1977.

[15] Kubin, L. P., Louchet, F., Peyrade, J. P., Groh, P., Cottu, J. P., Acta Metall. 27 (1979) 343. 